\title{
On the detection of myocadial scar based on ECG/VCG analysis
}

\author{
Sofia-Maria Dima ${ }^{1}$, Christos Panagiotou ${ }^{1}$, Evangelos B. Mazomenos ${ }^{2}$, James A. Rosengarten ${ }^{3}$, \\ Koushik Maharatna ${ }^{2}$, John Gialelis ${ }^{1}$, Nick Curzen ${ }^{3}$ and John Morgan ${ }^{3}$
}

\begin{abstract}
In this paper, we address the problem of detecting the presence of myocardial scar from standard ECG/VCG recordings, giving effort to develop a screening system for the early detection of scar in the point-of-care. Based on the pathophysiological implications of scarred myocardium, which results in disordered electrical conduction, we have implemented four distinct ECG signal processing methodologies in order to obtain a set of features that can capture the presence of myocardial scar. Two of these methodologies: a.) the use of a template ECG heartbeat, from records with scar absence coupled with Wavelet coherence analysis and b.) the utilization of the VCG are novel approaches for detecting scar presence. Following, the pool of extracted features is utilized to formulate an SVM classification model through supervised learning. Feature selection is also employed to remove redundant features and maximize the classifier's performance. Classification experiments using 260 records from three different databases reveal that the proposed system achieves $89.22 \%$ accuracy when applying 10fold cross validation, and $82.07 \%$ success rate when testing it on databases with different inherent characteristics with similar levels of sensitivity (76\%) and specificity (87.5\%).
\end{abstract}

Index Terms-Myocardial scar detection, ECG median beat, VCG, SVM, feature selection

\section{INTRODUCTION}

A Number of recent reports are indicative of the prevalence of ischemic heart disease (IHD) as the leading cause of death, both in developed and developing countries [1]. IHD can lead to the sudden interruption of the normal blood supply of the heart, clinically referred to as Myocardial Infarction (MI) or typically known as a heart attack. If proper blood flow is not restored, in a timely fashion, heart cells (myocardiocytes) near the area of the infarct die (necrosis) and result in scar tissues (fibrosis). These scar tissues have different electrical conduction properties than normal heart tissues, resulting in a disordered electrical conduction in the

This work was supported by E.U. ARTEMIS Joint Undertaking under the Cyclic and person-centric Health management: Integrated appRoach for hOme, mobile and clinical eNvironments - (CHIRON) Project, Grant Agreement \# 2009-1-100228.

1 S.M.Dima, C.Panagiotou and J.Gialelis are with the Industrial Systems Institute, ATHENA RC, Platani, Patras, 265 04. GR and Dep. of Electrical and Computer Engineering, University of Patras, 26500, GR (email: \{sdima,panagiotou,gialelis\}@isi.gr)

${ }^{2}$ E. B. Mazomenos and K. Maharatna are with the School of Electronics and Computer Science, University of Southampton, Southampton, SO17 1BJ, UK. (e-mail: \{ebm, km3\}@ecs.soton.ac.uk)

${ }^{3}$ J.A. Rosengarten, N. Curzen and J. Morgan are with the University Hospitals Southampton NHS Trust, SO17 1BJ, UK. (e-mail: james@rosengarten.co.uk, nick.curzen@suht.swest.nhs.uk,jmm@hrclinic.org

"Copyright (c) 2013 IEEE. Personal use of this material is permitted However, permission to use this material for any other purposes must be obtained from the IEEE by sending an email to pubs-permissions@ieee.org." heart. Such disordered conduction may potentially lead to fatal arrhythmias like Ventricular Tachycardia (VT) or Ventricular Fibrillation (VF) which are known to be the cause of sudden cardiac death (SCD). The risk of VF/VT occurrence is primarily defined by detecting the presence of scar tissue and estimating its size [2], [3]. Obtaining this information allows identification of the cases where the implantation of an Implantable Cardioverter-Defibrillator (ICD) device would be beneficial. Imaging techniques such as Contrast-Enhanced Cardiac Magnetic Resonance Imaging (CMR) can provide detailed information on the presence, location and extent of myocardial scar [4], [5]. However, in addition to its suitability only as a bedside test, CMR is a resource-demanding process, both in terms of cost and specialized personnel required for its operation. Additionally, other approaches for an accurate detection of scar are characterized as high interventional methods [6].

On the other hand, 12/15 lead Electrocardiogram (ECG) is widely available and can also be used in clinical as well as remote settings owing to its availability in the form of portable devices [7]. Since the ECG reflects the electrical conduction through the heart, it is expected that possible conduction anomalies, attributed to the presence of scar, would be directly imprinted on the ECG trace.

Therefore an automated ECG-based solution for myocardial scar detection deployable in fast and cost effective manner both in the primary-care and nomadic point-of-care may provide an effective tool for the initial screening of myocardial scar en masse. The high risk patients could then be subjected to a detailed CMR analysis and thereby optimizing the overall cost and resources. From this perspective, in this paper we explore the problem of effectively detecting the presence/absence of myocardial scar from standard ECG recordings.

The underlying idea is to investigate a set of ECG features obtained either directly from measurements or indirectly using signal processing techniques - which may be indicative to the anomalous conduction effect typical of myocardial scar and develop a supervised learning model capable of detecting its presence/absence. It's worth mentioning that the goal is neither to replace the existing scar detection modalities, nor to claim that the proposed method would be more accurate compared to the high-resolution imaging modalities, but to provide a tool that may differentiate the patients who need further rigorous clinical characterization so that the cost associated with diagnostic processes could be reduced and resource utilization could be done in optimal way.

In our investigation the feature set is constructed by includ- 
ing different ECG parameters already known to be associated with myocardial scar and then extending it with a number of new features that are also expected to be correlated with myocardial scar but have not been investigated before for scar detection. Four distinct strategies are followed for generating the feature set as follows:

- We introduce the concept of the "template-median ECG heart beat", produced from signal averaging of ECG heartbeats with confirmed absence of scar, on a per-lead basis. This median beat template is compared with the ECG signals that are under test based on a specific set of morphological features.

- A set of statistical features are computed from the ECG time series.

- A set of time-domain features of the ECG signals with known association to myocardial scar, e.g (durations, area under the ECG curve, presence of fractionated QRS etc...) are added in the feature pool.

- A set of spatial and vectorial features are estimated from the Vectorcardiogram (VCG).

In total 344 features are extracted, which are then subjected in a feature ranking process and the 25 most discriminating features are selected. From our exhaustive experiments, Support Vector Machine (SVM) provides the optimal solution for our classification system, which is tested in terms of accuracy (sensitivity and specificity) with 260 ECG signals from three different databases for which the presence/absence of scar and its size is recorded using CMR. The proposed classification system achieves a total accuracy of $89.22 \%$ (sensitivity $87.25 \%$ $91.18 \%$ specificity). The promising performance results indicate the potential of the proposed classification system as a non-invasive mechanism for the initial screening of the presence/absence of myocardial scar. Furthermore, given that the proposed system requires only standard 12/15-lead ECG, it can be effectively used as a risk-stratification scheme in situations where an immediate decision on the presence/absence of scar is needed but there is no availability of imaging modalities. The rest of the paper is structured as follows. Section II provides a background on the conduction effects caused by the presence of myocardial scar. The process of feature-space construction is described in Section III, while the derivation of the optimal features and the classifier formulation are covered in Section IV. Performance results from various classification experiments are discussed in Section IV and conclusions are drawn in Section V.

\section{BACKGROUND}

Scars are typically formed following an MI episode and the subsequent death of healthy heart cells. In the area where the heart cells die, a reactive process called fibrosis replaces them with a collagen connective tissue which forms the scar [8]. Myocardial scars create patches of delayed conduction regions separated by regions of normal heart tissues with normal conductive properties. Thus the probability of electrical desynchronization occurring during the cardiac cycle, leads to an increased possibility of fatal arrhythmias. In situations where the scar is located in the ventricles, a typical scenario after an MI episode, scar-related VT/VF can spontaneously occur, leading to hemodynamic collapse and SCD. It has been long established that these conduction abnormalities cause characteristic alterations on the ECG trace, such as prolonged $\mathrm{R}$-waves, change of the value of the QRS-angle, fractionations (notches, slurs) in the QRS-complex, elevation/depression of the ST-segment [9]-[11]. In addition there have been studies suggesting that the morphology of the T-wave (T-wave alternans) is affected by the presence of scar since it represents the repolarization of the ventricles [12]. Drawing inspiration for the potential of the ECG as a modality capable of detecting the presence of myocardial scar we have developed a novel system, based on supervised learning, presented in details in the following sections.

\section{Methodology}

In our approach, following the standard clinical practice, a single representative heartbeat per lead from standard 12/15lead ECG recordings is selected for analysis from each signal. The clinical philosophy behind this approach is that the presence of myocardial scar and hence the disordered conduction effect resulting from, is in general uniformly present in each heartbeat of the ECG/VCG tracings with very little variability. This is due to the fact that myocardial scar is a permanent damage of the myocardium which will consistently affect the propagation of the electrical signal during the operation of the heart regardless of the heart rate. A small variability (different QT, RR intervals, R-peak amplitude) may exist between different heartbeats in the same ECG trace because of the presence of noise, or breathing artefacts. However these variations from beat to beat are not significant except for the cases of performing different physical activities or developing rapid pathological conditions such as arrhythmic episodes. Since in the standard clinical practice 12/15-lead ECG is recorded with patients in standard positions, such variability is also minimal. Therefore selecting one beat from such a recording is expected to represent the actual clinical condition of the subject adequately. For each ECG recording used in this work the representative heartbeats are selected by three cardiologists, first blindly, and then reaching to a consensus.

In order to construct the feature space for our classification investigation we have followed four distinct signal processing strategies. Two of these are based on pure morphological analysis of ECG and VCG signals, one based on the statistical properties of ECG signal and the last one is based on formulating a median ECG beat as a template with which the similarity of the incoming ECG beat to be classified is analyzed. The overall approach of the features generation is depicted in Fig. 1 and each of the approaches are discussed in detail in the following subsections.

Before applying the feature extraction process in the preprocessing step, the ECG isoelectric line is removed using a series of three median filters as in a similar way as described in [13], [14] and all signals are resolved to the same resolution. We also used the TDMG algorithm [15] for detecting the ECG wave boundaries and peak that are used for feature extraction. 


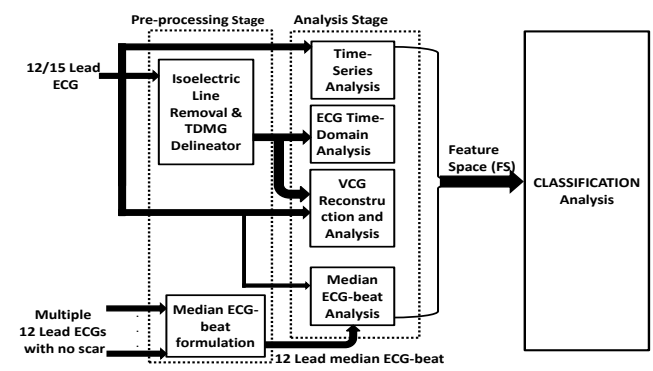

Fig. 1. Flowchart of the proposed Methodology
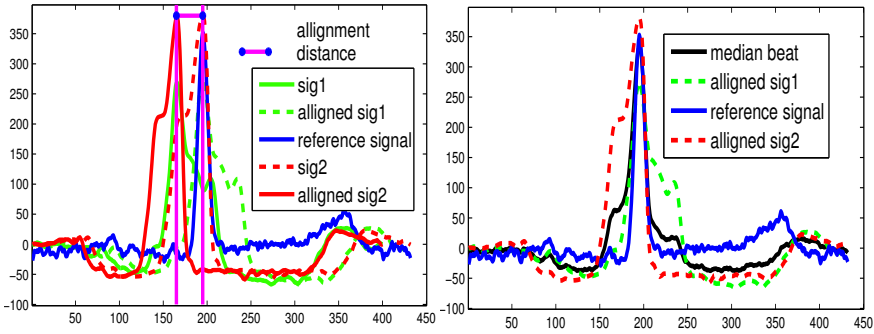

Fig. 2. Centralization and Alignment of incoming beats
Fig. 3. Extraction of the median beat

\section{A. Median-ECG beat analysis}

The concept of the median-ECG beat is introduced here as a novel approach for the detection of myocardial scar. The basic philosophy is to create a "template" ECG heartbeat vector - one beat per lead - for 12 ECG leads, that is free of any disordered conduction effect and thus considered representing condition of no-scar. Since disordered conduction is a signature that is present in the ECG of a scar patient (although the individual ECG lead capturing this effect could be different from person to person depending on the position and extent of scar), a per-lead based comparison of such heartbeats with the template should be able to capture the disordered conduction effect either through morphological dissimilarity or a phase difference between these two sets. In order to construct the median ECG template vector we selected ECGs with verified absence of scar (i. e. scar size 0\% in CMR) and additionally with absence of a set of confounders which may alter the ECG morphology in such a way resembling scar. Three cardiologists evaluated the ECG signals from one database (DB-I, see Section IV) blindly to each other and finally made a consensus on 38 12-lead ECG signals which could be considered to be "no-scar" ECG signals in clinical sense. Please note that although these ECGs represent no-scar or no confounding conditions, they may still have non-scar related QRS, ST or T wave changes compared to a normal ECG and therefore not necessarily are normal ECG beats. First, from each of these 38 records for each lead, one single heartbeat was isolated (again as per the consensus of the cardiologists). Ectopic beats are excluded in this process of formulation of the median beats. Then these selected beats from all the records are averaged on per-lead basis to construct the median beat vector. Mathematically this process can be described as:

$$
M_{i}=\operatorname{avg}_{j}\left(B_{i, j}\right)
$$

, where $M_{i}$ is the median beat for lead $i$ and $B_{i, j}$ is the isolated beat for lead $i$ and record $j, i \in 1,2, \ldots ., 12$ and $j \in 1,2, \ldots \ldots, 38$ and $\operatorname{avg}_{j}()$ is the averaging operation over the whole set of $j$. This operation creates the desired template median ECG beat vector $[M 1, M 2, \ldots ., M 12]$ which is used for final comparison on per-lead basis with each incoming 12lead beat that requires classification. However, the duration of a heartbeat in a specific lead may vary from person to person. To overcome this issue we first selected the isolated

beat of maximum duration for a particular lead $i$ - say, the reference beat - over all the records and detected its maximum deflection point (typically the R- or S-peak depending upon the specific lead under consideration $i$ - say, the reference point). The maximum deflection point of an isolated beat $B_{i, j}$ from the record $j$ for the same lead $i$ is first aligned to the maximum deflection point of this reference beat and then the whole beat $B_{i, j}$ is interpolated accordingly to make it of the same length as the reference beat. For example, if we have two signals for lead $i$, of 400 and 440 samples, and the R-peak is located in the 170th and 194th sample respectively, then the R-peak of the first signal has to be shifted 24 samples (by interpolating the first part from the beginning of the signal to the R-peak) and the remaining 16 samples have to be uniformly added to the second part of the signal (from the R-peak to the end of the beat) as shown in Fig. 2. This process is repeated for all the records to make all $B_{i}$, of equal duration for facilitating the averaging process as depicted in Fig. 3. Once the set of median beats is derived, they are compared on per-lead basis with the incoming ECG beats.

The comparative analysis between the median-ECG beat and the ECG beat under test takes place both in time and timefrequency domain. In the time-domain, the Cross-Covariance of these two time-series is calculated since it is a measure of similarity between two signals and is commonly used to find features of an unknown signal by comparing it to a known one. It is a function of the relative time between the two signals. Additionally, the cross-correlation which in essence is the normalized covariance is computed for determining timedelays in the propagation of electrical activity.

For the time-frequency domain analysis we have opted to investigate the Wavelet Coherence (WC) between the medianECG and the testing ECG, using the complex Morlet Wavelet (cmor1-0.5) as the basis function. It combines the concept of wavelet transform with the coherence analysis, quantifies the coherence and the phase synchrony between these two time series as a function of both time and frequency (scale) and is used to identify time and frequency intervals in which they have a strong correlation. The WC in our case is a complex product, where the level of coherence is shown by the magnitude of the WC, while the phase lag between the two signals is represented by the phase of the WC. The magnitude and the phase coherence is averaged over time and over the resolution scales.

As an example of the morphological alterations caused due 


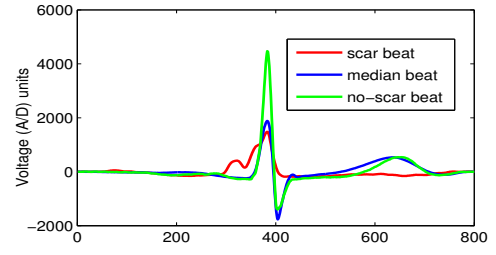

(a) (no-)scar beats vs. median beat

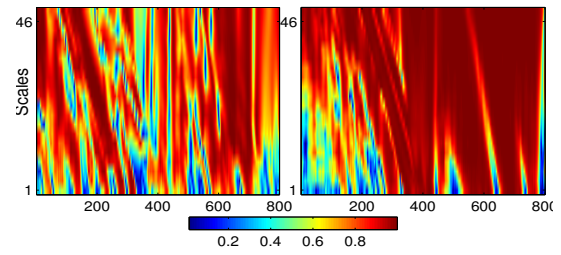

(b) Wavelet Magnitude Coherence (left:scar vs median, right:no-scar vs median)

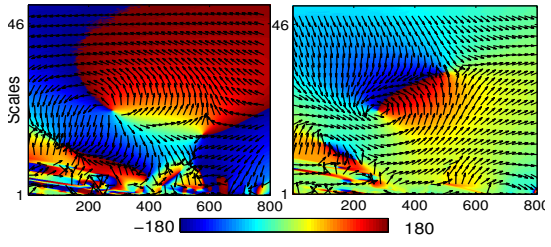

(c) Wavelet Phase Coherence (left:scar vs median, right:no-scar vs median)

Fig. 4. $\mathrm{WC}=\frac{\overline{C_{x}(a, b)} * C_{y}(a, b)}{\sqrt{\left|C_{x}(a, b)\right|^{2}} * \sqrt{\left|C_{y}(a, b)\right|^{2}}}, \mathrm{C}$ is the Wavelet transform, a,b: scales, location parameter

to delayed conduction from the presence of scar, Fig. 4(a) depicts the ECG trace of a record with scar and a record free of scar, alongside the constructed median-ECG beat for this particular lead. The fractionation in the QRS-complex as well as the prolonged QRS-width are obvious in the scarred signal. Furthermore, to illustrate the effect on the coherence levels, both signals are compared to the median-ECG beat in terms of modulus wavelet coherence and phase wavelet coherence. It is evident from Fig. 4(b) and Fig. 4(c), that there exist noticeably different levels of both magnitude and phase coherence for the two signals.

\section{B. Statistical parameters of the ECG timeseries}

Apart from the aforementioned morphological parameters a set of statistical features [16] on a per-lead basis have been computed which underpins the statistical property of an ECG signal. This set of statistical parameters is listed in TABLE I which also provides a brief description of possible significance of each feature in ECG analysis. In principle they provide effective means for analyzing the level of complexity and the type of distribution that a time-series exhibits. In the presence of scar tissues both the complexity and the distribution of the ECG trace will be affected, due to the alterations caused in the ECG morphology. Subsequently, it is expected that these parameters will capture these intricate changes which can then be used as an indication of scar presence.

Apart from these parameters since ECG's fluctuations could be non-random and in essence a reflection of the dynamics of the cardiovascular systems [19], measuring fluctuations in the frequency and time domain may reveal significant information on the dynamic characteristics, which are lost with routine averaging or linear spectral methods. According to this observation, the Detrended Fluctuation Analysis (DFA) is used to quantify the fractal scaling properties and to determine the self-affinity of the heartbeat.

\section{Time domain ECG parameters}

Various studies have described possible changes in QRScomplex and T-wave morphology in an ECG trace due to the presence of myocardial scar. Drawing from our experience in Cardiology primary care in consultation with available literatures, the ECG morphological features showing the effect of disordered conduction more prominently, are the QRS duration, the T-duration and the T-peak to T-offset duration. We
TABLE I

PhySiCAL MEANING OF STATISTICAL PARAMETERS

\begin{tabular}{|l|l|}
\hline Statistical parameter & Physical Meaning \\
\hline Shannon entropy & $\begin{array}{l}\text { Measure of randomness of } \\
\text { the ECG signal }\end{array}$ \\
\hline Hurst parameter [17] & $\begin{array}{l}\text { Measure of long-range de- } \\
\text { pendence, indicative of non- } \\
\text { stationary nature, increase } \\
\text { in complexity and changes } \\
\text { in space-filling properties of } \\
\text { the ECG signal }\end{array}$ \\
\hline Hjorth Complexity/ Hjorth Mobility [18] & $\begin{array}{l}\text { Measure of mean power } \\
\text { ratio of higher frequency } \\
\text { energy over the total energy } \\
\text { of the ECG signal. Indica- } \\
\text { tive of the signal's complex- } \\
\text { ity }\end{array}$ \\
\hline Median value, interquartile range & $\begin{array}{l}\text { Index of the distribution of } \\
\text { the ECG signal }\end{array}$ \\
\hline Skewness & $\begin{array}{l}\text { Measure of the asymme- } \\
\text { try of the probability distri- } \\
\text { bution of the ECG signal, } \\
\text { more specifically, the QT in- } \\
\text { terval }\end{array}$ \\
\hline Kurtosis & $\begin{array}{l}\text { Index of how much peaked } \\
\text { the ECG signal is around } \\
\text { its mean, may characterized } \\
\text { QT interval }\end{array}$ \\
\hline Mean, standard deviation, variance & $\begin{array}{l}\text { Index of the average (ex- } \\
\text { pected) value and the vari- } \\
\text { ation/dispersion of the ECG } \\
\text { signal }\end{array}$ \\
\hline & \\
\hline
\end{tabular}

first derive these features on per-lead basis and also calculate the RMS and dispersion (maximum-minimum) values of these, using all 12 leads. In addition, in order to capture potential morphological changes in these waves more accurately, we calculate the area under the curve of the QRS-complex and the T-wave, using trapezoid integration with the isoelectric line as the reference. It has also been shown in literature that presence of fractionations (notches, slurs) in the QRS complex is a potential manifestation of disordered conduction effect. Therefore we include the total number of fractionations present in the QRS and a binary value indicating the presence/absence of fractionation in the terminal QRS deflection as two separate features. We follow the definitions provided in [14] for identifying the fractionations present in the QRS complex. Finally, a binary parameter that indicates whether or not, the QRS-offset (j-point) is elevated by more than $0.1 \mathrm{mV}$ is also calculated. The final type of feature we computed is the spectral energy of the QRS-complex and the T-wave using Discrete Wavelet 
Transform (DWT).

\section{VCG based features}

The electrical impulse that is propagated along the heart regions can be described from the magnitude and the direction of the electrical forces that the heart generates. The magnitude and direction of these forces form a resultant vector that evolves in time on the 3-D space around the origin [20]. The continuous recording of the electrical potentials (resultant's vector's end points), presented in the 3-D space, generates curved lines (loops) along two or three perpendicular axes and it is known as the VCG (Fig. 5). In contrast, the ECG represents the electrical potential only on a single axis.

The predominant method for the recording of the VCG is attributed to Frank [21]. Frank's XYZ leads consist of three leads derived from seven electrodes and represent the right-left axis (X), head-to-feet axis (Y) and frontback (anteroposterior) axis ( $\mathrm{Z}$ ) which constitute the three components of the resultant vector. In clinical practice there is poor utilization of the VCG, thus the recordings from Frank's orthogonal leads are quite rare. In principle each lead of the standard 12-lead ECG, can be reconstructed from Frank's XYZ lead. Therefore, Frank's XYZ leads can also be reconstructed from 12-lead ECG recordings. Among all the approaches of reconstructing the ECG from VCG and vice versa, Dower's approach [22] is the most clinically accepted method and is adopted for constructing the VCG from a 12-lead ECG. Dower's matrix consists of lead specific coefficients that are used to calculate the eight independent leads (V1-V6, I and II) as a linear combination of the VCG. Subsequently, the inverse process produces the VCG leads from the 12-lead ECG. The $3 \times 8$ inverse Dower matrix (iD) is given as:

$$
\begin{gathered}
i D=\left[\begin{array}{ccccccccc}
-0.172 & -0.074 & 0.122 & 0.231 & 0.239 & 0.194 & 0.156 & -0.010 \\
0.057 & -0.019 & -0.106 & -0.022 & 0.041 & 0.048 & -0.227 & 0.887 \\
-0.229 & -0.310 & -0.246 & -0.063 & 0.055 & 0.108 & 0.022 & 0.102
\end{array}\right] \\
L=\left[\begin{array}{ccccccc}
V 1 & V 2 & V 3 & V 4 & V 5 & V 6 & I I
\end{array}\right]^{T}, V C G=\left[\begin{array}{llll}
V C G x & V C G y & V C G z
\end{array}\right]^{T} \\
V C G=L \times i D
\end{gathered}
$$

In our work, we employed the recorded VCG, if available, and used the inverse Dower transform where recorded VCGs were not available. As it is already mentioned, the conduction delays and disordered heart activity, caused by myocardial scar, are reflected on the ECG as fractionated QRScomplex and/or prolonged R-wave and/or alterations on $\mathrm{T}$ wave's morphology. Since the VCG is a linear combination of the ECG leads we expect that these morphological alterations will also be reflected on the VCG's spatial characteristics Fig. 5. The QRS-complex and T-waves boundaries, extracted using TDMG [15], allowed the localization of these waves in the VCG loop. Also the temporal position of the R- and Tpeak allowed the construction of the R- and T-peak vectors. Additionally we constructed the vectors that represent the maximum width of the QRS- and T-loop respectively. For these four vectors we calculated the magnitudes and angles for each of the three 2-D projections(X-Y, X-Z, Y-Z) of the 3-D VCG. The areas for each of the 2-D projections were extracted
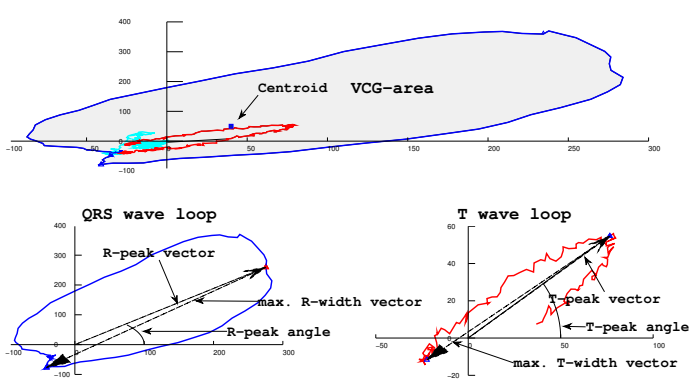

Fig. 5. Illustration of the VCG Feature Set (FS-III)
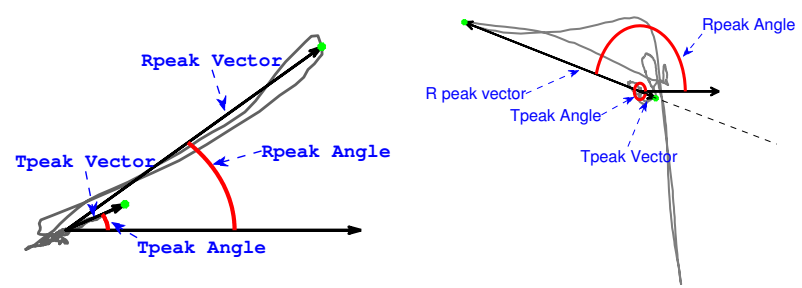

Fig. 6. R- and T-peak angle in No-Scar(left) and Scar(right) VCG

by calculating the number of pixels that each of the two waves enclose. Finally the centroid point - defined as $C=\frac{\int x g(x) \mathrm{dx}}{\int g(x) \mathrm{dx}}$ - is calculated and the respective vector of the VCG loop was constructed, since it is expected to be affected by the VCG morphological alterations. The feature dataset was further fed with the magnitude and angle of the centroids' vectors. An indicative example of how the scar presence is reflected to the VCG's spatial features is depicted in Fig. 6, where the R- and T-peak angles are significantly different for scar and no-scar records.

TABLE II summarizes all the features considered in this work. Given the inherent variability in ECG signals among patients, each of these feature spaces offers information that

\begin{tabular}{|c|c|c|}
\hline \multirow[b]{2}{*}{ is } & $\begin{array}{c}\text { median analysis } \\
\text { metrics }(1)\end{array}$ & $\begin{array}{l}\text { cross correlation } \\
\text { covariance } \\
\text { wavelet magnitude \& phase coherrence }\end{array}$ \\
\hline & $\begin{array}{l}\text { statistical timeseries } \\
\text { metrics (2) }\end{array}$ & $\begin{array}{l}\text { Mean, Variance \& Standard Deviation values } \\
\text { Median value \& Interquartile Range } \\
\text { Entropy } \\
\text { Kurtosis \& Skewness } \\
\text { Hjorth Mobility \&Complexity } \\
\text { Hurst Exponent } \\
\text { Detrended Fluctuation analysis }\end{array}$ \\
\hline $\begin{array}{l}\text { 䒚 } \\
\text { I }\end{array}$ & $\begin{array}{l}\text { ECG time domain } \\
\text { metrics(3) }\end{array}$ & $\begin{array}{l}\text { J point elevation/depression } \\
\text { Fractionations in terminal deflection } \\
\text { QRS/T rms \& dispertion width } \\
\text { Tpeak-Toffset interval (per lead \& rms) } \\
\text { QRS/T wave width,energy \& area } \\
\text { Fractionations in QRS }\end{array}$ \\
\hline $\begin{array}{l}\text { 目 } \\
\text { 至 }\end{array}$ & $\begin{array}{l}\text { VCG spatial } \\
\text { metrics(4) }\end{array}$ & $\begin{array}{l}\text { VCG area } \\
\text { R-width, T-width magnitude } \\
\text { R-peak, T-peak \& Centroid vector magnitudes } \\
\text { R-peak T-peak \& Centroid vector angles }\end{array}$ \\
\hline
\end{tabular}

TABLE II

Complete Feature SeT 
can possibly be used to distinguish between the presence and absence of scar.

It is to be noted that while constructing the feature space we did not consider the location of scar. The main reason behind this is, due to the surface recording nature of ECG the signature of scar on a lead is manifested as a complex interaction between its location and extent. Therefore any attempt in trying to localize scar from ECG only, is not encouraged in clinical practice as it may not give clinically acceptable result. On the other hand, since each ECG lead captures a different spatial view of the heart, it is expected that depending on the location and extent of the scar, its overall effect will be manifested in one or more leads. Therefore with our adopted strategy of analysis in a per-lead basis it should be possible to capture the overall effect of the presence of scar irrespective of its location.

\section{Classification Strategy and Validation}

\section{A. Databases}

The above mentioned parameters are then used to formulate a robust classification model, able to discriminate the presence/absence of myocardial scar, following a supervised learning approach. In our classification investigation we utilized ECG records from three databases, two of them (DBI and II) constructed during clinical evaluation of patients at the Cardiology Department of the University Hospital Southampton NHS Trust. These constitute of patients which had 12-lead ECGs and CMR measurements performed within the same period. Standard 12-lead ECG recording was done by trained cardiographer with the patients in a supine position. The CMR result provides also the size (in terms of \%) of the scarred tissue. For our classification purposes any scar size $>0 \%$ is considered as presence of scar. It is worth noting that the CMR resolution is also limited and therefore some of the smaller scar sizes could be considered as $0 \%$ scar in its analysis. However CMR is a standard procedure that is clinically accepted and employed in clinical practice on a daily basis and appropriate clinical action is taken based on its outcome. Therefore CMR analysis is widely used as a clinical standard for assessing the extent of scar in real life. Given this fact we have used CMR outcome as the ground truth for assessing the extent of scar.

In order to balance our dataset as much as possible we have included a third database (DB-III) containing ECGs with no scar presence. This was obtained from the 52 healthy control records of the PTB Diagnostic ECG Database (DB-III), publicly available in Physionet [23]. Although DB-III offers no CMR measurements, the annotation of these records as healthy controls, allowed us to imply that no scarred tissue is

TABLE III

CHARACTERISTICS OF THE DATABASES

\begin{tabular}{|c|c|c|c|c|}
\hline Database & \multicolumn{2}{|c|}{ Nature of records } & Sampling frequency(Hz) & VCG \\
\hline & Scar & No-Scar & & \\
\hline DB-I & 108 & 46 & 500 & Reconstructed \\
\hline DB-II & 50 & 4 & 1000 & Reconstructed \\
\hline DB-III & 0 & 52 & 1000 & Recorded \\
\hline
\end{tabular}

present. The frequency and the resolution are the same as (DBII). DB-III includes measurements of Frank's XYZ orthogonal leads, so the VCG was directly obtained without the need for reconstruction through iD. From all available records we isolated a single heart-beat and used it in our analysis. Expert cardiologists ensured that from the records that had scar no ectopic beat was selected. In total 260 records were considered with 158 records having CMR verified scarred tissue. The fundamental characteristics of the databases are summarized in TABLE III.

Although it is conceivable that DB-III is mainly taken from the public available database and thus may be debatable in terms of its utility regarding the actual clinical conditions of myocardial scar, DB-I and II contain data that are prospectively collected by expert clinicians and these two databases are in true sense representative of clinical patients since they are collected from those under investigation of myocardial scar at the Cardiology Department of the Southampton University Hospital NHS Trust. One important issue here is that, as mentioned earlier, presence of certain types of confounders may be manifested in ECG in the same way as the scar. It is for this reason that we did not exclude patients with these confounding ECG changes, and that accurate classification despite these ECG confounders was an important aim of this work.

\section{B. Classification Model}

The classification algorithm that has been utilized in this work is the Support Vector Machine(SVM) [24]. The analysis was facilitated by employing the Weka machine learning software, which implements the Sequential Minimal Optimization algorithm (SMO) for solving the quadratic programming optimization problem that arises when determining the maximum margin hyperplane of the SVM classifier. Several experiments with Gaussian and polynomial SVM, and with different configuration parameters were conducted, until an optimal setup was found. The performance evaluation metrics we present in our experiments are the classification accuracy, the specificity and the sensitivity as follows: sens $=\frac{T P}{T P+F N}$, spec $=\frac{T N}{T N+F P}, a c c=\frac{T P+T N}{F N+F P+T P+T N}$ Where, True Positive(TP)/False Negative(FN) the records that have scar and are correctly/incorrectly identified, while False Positive(FP)/True Negative (TN) the records that do not have scar and are incorrectly/correctly classified.

For building the classification model two particular issues were considered as fundamental requirements: minimization of possible overfitting and consistency of the three evaluation metrics - sensitivity, specificity and accuracy - between sequential experiments. The overfitting problem is common in classification schemes and is typically apparent when the classification model trained on a dataset fails to give satisfactory result for another arbitrarily chosen dataset. It may occur due to a) presence of noise or redundant features in the feature vector and b) due to existence of irrelevant patterns resulting from inherently error prone data entry and acquisition process. Subsequently the irrelevant input features should be excluded from the feature vector in order to produce a desired learning 
TABLE IV

EVALUATION RESULTS OF THE PROPOSED METHODOLOGY

\begin{tabular}{|c|c|c|c|c|c|c|c|c|c|}
\hline Metric & \multicolumn{8}{|c|}{ Performance\% } & Details \\
\hline & FS-I & FS-II & FS-III & RFS-I & RFS-II & RFS-III & $\overline{\mathbf{A F S}}$ & RAFS & \multirow{5}{*}{$\begin{array}{l}\text { 10-runs } 10 \text {-fold CV } \\
\text { Balanced DB-I }\end{array}$} \\
\hline Exp. & & I & & & II & & $\mathrm{V}$ & VI & \\
\hline Acc. & $63.04 \%$ & $65.21 \%$ & $69.56 \%$ & $84.78 \%$ & $81.52 \%$ & $71.45 \%$ & $61.95 \%$ & $91.3 \%$ & \\
\hline Sens. & $65.22 \%$ & $63.04 \%$ & $71.74 \%$ & $86.96 \%$ & $89.13 \%$ & $73.91 \%$ & $60.87 \%$ & $91.3 \%$ & \\
\hline Spec. & $60.87 \%$ & $67.39 \%$ & $67.39 \%$ & $82.61 \%$ & $73.91 \%$ & $63.04 \%$ & $63.04 \%$ & $91.3 \%$ & \\
\hline Exp. & & & & & III & & & $\begin{array}{l}\text { VII } \\
\end{array}$ & \\
\hline Acc. & & & & $71.7 \%$ & $78.3 \%$ & $85.85 \%$ & & $82.07 \%$ & Train with balanced DB-I \\
\hline Sens. & & & & $74 \%$ & $56 \%$ & $88 \%$ & & $76 \%$ & Test on DB-II,DB-III \\
\hline Spec. & & & & $69.6 \%$ & $98.21 \%$ & $83.93 \%$ & & $87.5 \%$ & \\
\hline Exp. & & & & & IV & & & VIII & \\
\hline Acc. & & & & $84.31 \%$ & $83.33 \%$ & $82.36 \%$ & & $89.22 \%$ & 10-runs 10 -fold CV \\
\hline Sens. & & & & $87.25 \%$ & $86.27 \%$ & $84.31 \%$ & & $87.25 \%$ & Balanced DB-I/II/III \\
\hline Spec. & & & & $81.37 \%$ & $80.39 \%$ & $77.36 \%$ & & $91.18 \%$ & \\
\hline Exp. & & & & & & & & IX & \\
\hline Acc. & & & & & & & & $69.05 \%$ & Train Balanced DB-I \\
\hline $\begin{array}{l}\text { Sens. } \\
\text { Spec. }\end{array}$ & & & & & & & & $\begin{array}{l}59.82 \% \\
87.5 \%\end{array}$ & Test on the remaining of DB-I and DB-II/III \\
\hline Exp. & & & & & & & & $\mathrm{X}$ & \\
\hline Acc. & & & & & & & & $91.3 \%$ & LOO \\
\hline Sens. & & & & & & & & $91.3 \%$ & Balanced DB-I \\
\hline Spec. & & & & & & & & $91.3 \%$ & \\
\hline Exp. & & & & & & & & XI & \\
\hline Acc. & & & & & & & & $87.25 \%$ & LOO \\
\hline Sens. & & & & & & & & $87.3 \%$ & Balanced DB-I/II/III \\
\hline Spec. & & & & & & & & $87.3 \%$ & \\
\hline
\end{tabular}

(R)FS -I -II -III: (Reduced) Feature Space from Median-, Time-, VCG-domain (R)AFS: (Reduced) All domains Feature Space

result and avoid the learning algorithm to overfit to the noise. Owing to the relatively small size of the database and the high dimensionality of the produced feature set in our case, to identify the most informative feature set capable of identifying presence or absence of scar, we ran extensive feature selection experiments using the algorithms like, Information Gain, Relief, SVM Attribute Evaluation (SVMAttributeEval), and based on their performance SVMAttributeEval was chosen. SVMAttributeEval is implemented with the Ranker search method which evaluates the importance of a feature by using an SVM classifier. Features are ranked according to the square of the weight assigned to them by the SVM. After excluding the redundant features the model is iteratively trained with the reduced feature set. This process is expected to reduce the possible overfitting as in principle the redundant features are eliminated from the original feature set. In order to evaluate the efficiency of the system and to identify the degree of overfitting we employed the traditional approach of 10 -fold Cross-Validation (CV) 10 times. The generated model was evaluated in terms of accuracy, sensitivity and specificity over a number of carefully constructed experiments to check the consistency of the three above mentioned performance parameters. In addition, the generated model trained with one database is applied on the other databases with inherently different characteristics to ascertain the effect of possible overfitting. Furthermore, we employed the Leave One Out (LOO) Cross Validation, which is a K-fold cross-validation strategy, $\mathrm{K}$ being equal to the number of instances in the original dataset, to evaluate the efficiency of our model. In summary, our experimental strategy uses the 10-times of 10 -fold CV as the basic experiment, which gives a good estimation of the classification performance of the generated model. In case the model does not satisfy the predictability (i.e. low accuracy) there is no reason to continue with the other testing experiments, as the basic classification capability of the system does not exist in this case. Otherwise, it is tested with other databases so as to investigate the capability of the model to classify unknown instances correctly and to estimate the possibility that the model has been fitted with features that are irrelevant to the myocardial scar. The consistency of the evaluation metrics and their comprehensive analysis establishes the predictability, robustness and reliability of the classification model.

\section{Analysis of Results and Model Performance Evaluation}

The overall results of our experiments are shown in TABLE IV. As presented in TABLE II we have four subsets of features. Initially we merge the first two subsets into one feature space FS-I, while the two remaining subsets consist of feature space II \& III (FS-II and FS-III) respectively. The total number of parameters is 192,125 and 27 for FS-I, II, and III respectively. At first (Exp.-I in TABLE IV), a balanced dataset from DB-I is constructed, using 46 records without scar tissues and 46 records with the highest scar size. On this database 10 runs of 10-fold $\mathrm{CV}$ was performed using the features from FS-I, II, and III independently without invoking any feature selection algorithm. As it is shown, the performance results are not sufficient enough to achieve an acceptable classification rate. This leads us to believe that there exist significant redundancies in all the three feature spaces. Therefore a feature selection step is carried out for each of the feature spaces using the SVMAttributeEval algorithm iteratively and observing the classification performance at each iteration step. Specifically, the output of each iteration of the feature selection algorithm 
TABLE V

Selected Parameters BASEd on the SVMAtTRIBUteEval

\begin{tabular}{|c|c|c|c|}
\hline RFS-I (Median \& Statistical) & RFS-II (Time Domain) & RFS-III (VCG) & RAFS \\
\hline Variance lead aVR & T-wave area lead aVR & T-wave angle $\mathrm{XY}$ projection & T-wave area lead I \\
\hline covariance lead aVR & Area of QRS-wave lead II & R-wave angle at XYprojection & wavelet magnitude coherence lead V4 \\
\hline Kurtosis lead V4 & T-wave area lead I & T peak vector magnitude YZ projection & T-wave angle $\mathrm{XY}$ projection \\
\hline wavelet magnitude coherence lead V4 & QRS-wave area lead aVR & T-width magnitude $\mathrm{XY}$ projection & Variance of lead aVR \\
\hline Entropy lead II & T-width lead V2 & R-wave angle YZ projection & QRS width lead aVF \\
\hline Mean Value lead V9 & T energy lead II & T-width magnitude YZ projection & Hjorth Mobility lead II \\
\hline Standard Deviation lead V4 & T-wave area lead V5 & T peak vector magnitude $\mathrm{XY}$ projection & Fractionations in terminal deflection lead V2 \\
\hline Hurst Exponent lead I & T-wave area lead aVR & VCG area YZ projection & $\mathrm{T}$ wave area lead aVR \\
\hline wavelet phase coherence lead aVF & T-wave area lead V3 & R-wave angle $\mathrm{XZ}$ projection & wavelet phase coherence lead aVF \\
\hline Interquartile Range lead aVR & T-wave area lead II & $\mathrm{R}$ peak vector Magnitude at $\mathrm{YZ}$ projection & Entropy lead II \\
\hline Hurst Exponent lead V1 & QRS-width lead aVF & & QRS energy lead V3 \\
\hline Standard Deviation lead V2 & QRS-width lead V6 & & Fractionations in terminal deflection lead V3 \\
\hline wavelet phase coherence lead V6 & QRS-width lead V2 & & Kurtosis lead V4 \\
\hline wavelet magnitude coherence lead aVR & QRS-wave area lead V1 & & cross-correlation lead III \\
\hline Cross-correlation lead III & T-width lead V6 & & T-width lead II \\
\hline Hjorth Mobility lead II & T-width lead II & & wavelet magnitude coherence of lead avR \\
\hline wavelet phase coherence lead aVL & T-wave area lead V4 & & Fractionations in QRS lead aVF \\
\hline covariance lead aVF & QRS-wave energy lead V3 & & covariance lead aVF \\
\hline Standard Deviation lead V5 & Fractionations in QRS lead aVL & & Mean Value lead V3 \\
\hline Kurtosis lead V4 & T-wave energy lead aVR & & Hurst Exponent lead V1 \\
\hline wavelet phase coherence lead II & QRS dispersion & & T-wave energy lead V2 \\
\hline Kurtosis lead III & T energy lead aVR & & Hjorth Mobility lead aVR \\
\hline wavelet phase coherence lead V1 & Fractionations in QRS lead aVF & & T-wave energy lead aVR \\
\hline Interquartile Range lead III & Fractionations in QRS lead V5 & & wavelet phase coherence lead V6 \\
\hline Hjorth Mobility lead aVR & T energy lead V2 & & VCG area YZ projection \\
\hline
\end{tabular}

(different number of selected features for each iteration), was the input used to train our model. At the end of this exhaustive simulation procedure, 25 features from each of FS-I and FS-II, and 9 features from FS-III achieved maximum performance. These optimal feature sets corresponding to FS-I/II/III are designated as RFS-I/II/III respectively and are listed in the first three columns of TABLE V. Using the same dataset (DBI) as before, 10 runs of 10 -fold $\mathrm{CV}$ results in classification accuracy of $84.78 \%, 81.52 \%$ and $71.45 \%$ in RFS-I, II and III respectively (Exp-II in TABLE IV). In particular, the sensitivity and specificity in RFS-I are also high $(86.96 \%$ and $82.61 \%$ respectively) showing its capability for simultaneously identifying ECGs with and without scar accurately. Therefore the models generated during the training phase of Exp-II have been applied on DB-II and III (Exp-III) to check the robustness of the model and existence of possible overfitting. It was found that the performance of the model is poorer in terms of all the three metrics in RFS-I and II (except specificity in RFS-II) whereas it showed good performance in RFS-III. However the conflict between the performance results in RFS-III in Exp. II and III imposed further investigation into the feature space. In the last experiment, where the three feature spaces were investigated separately, we merged the records from all the three DBs and constructed a balanced dataset with equal number of scar and no scar records (Exp-IV). As the available number of no-scar records is 102 (the remaining 158 records have scar tissue), we construct a dataset of 204 instances, and applied 10 runs of 10 -fold cross validation on this dataset for each RFS. In this case, all the RFS showed comparable results although the specificity in RFS-III is slightly lower than the other two.

The inconsistency between the results of the above mentioned experiments carried out on separate feature spaces prompted us to believe that either the feature sets are not enough descriptive or a degree of overfitting exists. Therefore we merged the parameters from the three feature spaces into one pool of features (All Features Space-AFS) and applied 10 runs of 10 -fold cross validation (Exp-V) without feature selection step. The poor results indicate towards existence of significant redundancies.

Following the same strategy as before we perform feature selection in the AFS feature space, producing the Reduced All Features Space (RAFS) consisting of 25 features as shown in the fourth column of TABLE V. Within these 25, six features came from the comparison of the testing ECG with the median beat, two of them are spatial characteristics from the VCG domain, eight of them are from the time domain analysis and the remaining parameters are features from the time series analysis. The importance of these parameters is shown in a 2D scatter plot between the T-area (lead I) and Wavelet modulus Coherence (lead V4)- the top two parameters in the feature ranking- in Fig. 8, where one may see the formulation of two clusters (scar/no-scar records). It is important to observe that the consistency of the features is demonstrated by the fact that the majority of the dominant features of the RAFS feature space, although maybe generated from a different lead (which is typical in standard clinical practice and may be attributable to the spatial location and extent of the scar since different ECG leads are expected to capture effects from different regions of heart), are also present in the list of the dominant features of the individual feature spaces. This fact verifies the significance of these particular features regardless of the feature space to provide discriminative properties for the detection of the presence/absence of myocardial scar. These dominant features are highlighted in TABLE V.

Utilizing the feature vector of RAFS we ran repeated 10fold $\mathrm{CV}$ in Exp-VI. The results show that high classification rate of $91.3 \%$ is achieved. In addition, the model achieved significant level of sensitivity and specificity indicating that the model is able to discriminate the presence/absence of significant myocardial scar. However, as the training set used 


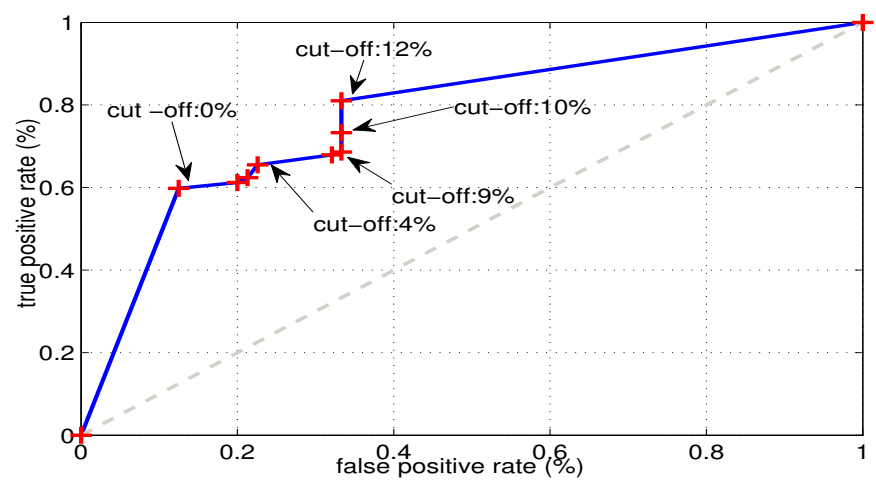

Fig. 7. Cross validation results using different cutoff sizes
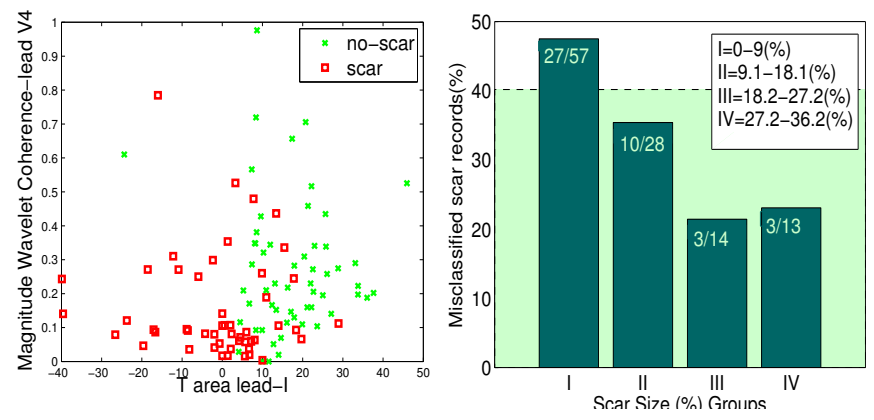

Fig. 8. Scatter Plot of T-area (Lead I) Fig. 9. Percentage of misclassified \& Magnitude WC V4 scar records vs scar size

only instances from the DB-I, we tested the model on instances that came from different sources as in the presence of possible overfitting, in this case, the classification behavior of the model is expected to be markedly different. Exp-VII is carried out from this point of view where we used DB-II and III as the testing sets which are inherently different from the characteristics of DB-I. The overall accuracy achieved is $82.07 \%$, while the specificity is in the same high levels (Exp-VII). Moreover, it is established in [25] that a model is robust enough if its behavior doesn't change significantly when it is fed with new data. This is why we applied Exp. VIII, where we merge all the available instances from the three databases and we construct a balanced dataset of 204 instances (all no-scar records and 102 scar records), using all features from the RAFS. Repeated 10 -fold cross-validation results in this experiment also shows consistency with Exp VI and VII indicating that the model provides classification performance $(89.22 \%$ with similar high level of specificity and sensitivity values) with high degree of confidence. Since the Leave-One-Out process is considered as good indicator of a system's robustness [26], we applied that strategy on the setup of Exp. VI and Exp. VIII and executed two additional experiments Exp. $\mathrm{X}$ and Exp. XI, which once again show consistent performance with high level of accuracy, sensitivity and specificity. Summarizing the results of all the experiments in addition to the adopted standard processes of reducing overfitting in the model building phase, it is apparent that although there is possibility of existence of overfitting, it is minimal.

We further carried out an extension of Exp-VII in ExpIX by adding the remaining records from DB-I as testing records. As the results show, the accuracy and sensitivity are decreased (69.05\%), while the specificity remains at the same levels. Further investigation revealed that the drop of classification rate in this experiment may be attributable to the misclassification of the lower scar size records. As it is illustrated in Fig. 9 the number of False Negatives decreases as the scar size increases. More specifically, we observed that when the scar size exceeds the threshold of $9.8 \%$ our model's sensitivity reaches to $81.4 \%$. To explore this further we created the ROC curve, where the Exp-IX is repeated by keeping the training model the same but refining the class label only for the testing set, according to a tuning cut-off scar size as shown below (Fig. 7). It is evident that as the labeling of the scar size cut-off increases to $>10 \%$, both the sensitivity and consequently the classification accuracy increases appreciably, while the specificity remains at the same levels whereas for lower scar cut-offs the sensitivity ranges between $60-75 \%$. This fact leads to the conclusion that the model can faithfully distinguish between significant scarring and no-scar whereas it is comparatively less accurate for lower scar size.

The evaluation results presented so far are based on the $>0 \%$ scar size as the definition of presence of scar. We further investigated the system's performance by changing this threshold and then retraining and reapplying the entire system in order to accommodate possible resolution issues of CMR measurement. The performance of the system is explored considering different cut off values $(0 \%-10 \%)$. This analysis is conducted using the setup of Exp. VIII, where a balanced dataset is constructed between the two classes and the 10-fold $\mathrm{CV}$ process is applied for estimating the performance. The resulting ROC curves are depicted in Fig. 10. From the ROCs comparison we observe that the $0 \%$ threshold demonstrated the best performance as it is located near the left upper edge and the area under the curve (AUC) is the maximum among all the other cases. The obtained performance resulting from the experiments in the RAFS space confirms that the signal processing techniques that we have followed can indeed provide a set of features able to discriminate the presence of myocardial scar from ECG recordings, particularly when the scar size is significant - a condition where immediate clinical attention is needed.

However the model proposed here should be considered as

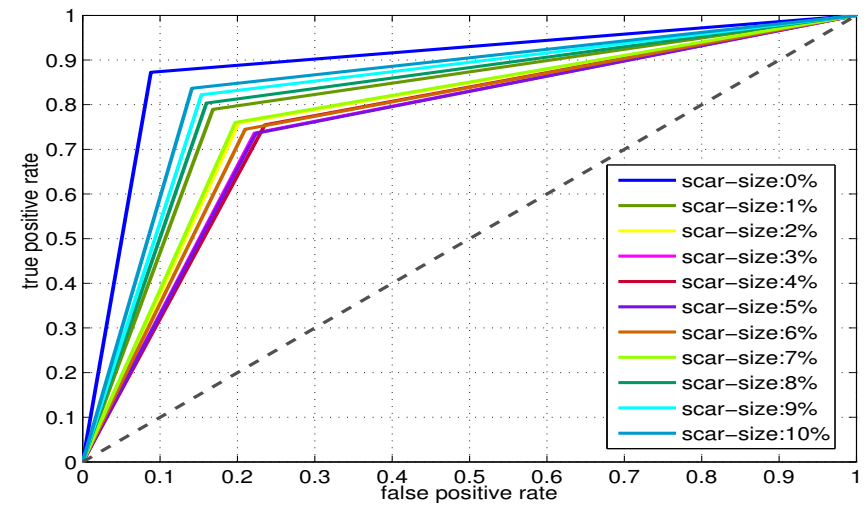

Fig. 10. Cross validation results using different cutoff sizes 
a promising first-step result for classifying myocardial scar from ECG and not as a completely ready method for clinical translation. This is due to the retrospective nature of the experiments conducted. The accuracy, sensitivity, specificity and the possible extent of existing overfitting need to be evaluated under a prospective trial which is planned in the future but currently beyond the scope of the present work.

\section{Conclusions}

In this paper we have presented the development of a classification scheme, for the detection of myocardial scar from ECG and VCG recordings. Four different strategies have been explored so as to obtain a set of features that will enable the discrimination between scar presence and absence. The application of dimensionality reduction along with the use of the SVM classifier, formulated a classification strategy that achieves $89.22 \%$ classification accuracy, as evaluated in $\mathrm{CV}$ experiments. The robustness of our proposed model is also justified in experiments where the training and testing sets are from different databases, in which the proposed system maintains the high performance, achieving an $82.07 \%$ overall accuracy with similar levels of sensitivity (76\%) and specificity $(87.5 \%)$. The obtained performance results reveal the potential of the proposed classification methodology to provide an efficient tool for the early screening in the pointof-care of myocardial scar in the point-of-care using costeffective, ubiquitous ECG/VCG recordings.

\section{REFERENCES}

[1] V. L. Roger et al., "Executive summary: Heart disease and stroke statistics-2012 update: A report from the american heart association," Circ., vol. 125, no. 1, pp. 188-197, 2012.

[2] D. Bello et al., "Infarct morphology identifies patients with substrate for sustained ventricular tachycardia," J Am Coll Cardiol, vol. 45, no. 7, pp. $1104-1108,2005$.

[3] A. T. Yan et al., "Characterization of the peri-infarct zone by contrastenhanced cardiac magnetic resonance imaging is a powerful predictor of post-myocardial infarction mortality," Circ., vol. 114, no. 1, pp. 32-39, 2006.

[4] M. Ranji, et al., "Quantifying acute myocardial injury using ratiometric fluorometry," IEEE Trans. Biomed. Eng, vol. 56, no. 5, pp. $1556-1563$, may 2009.

[5] P. Scott et al., "Left Ventricular Scar Burden Specifies the Potential for Ventricular Arrhythmogenesis: An LGE-CMR Study." J. Cardiovasc. Electr, vol. 24, no. 4, pp. 430-436, Oct 2012.

[6] A. Porras et al., "Interventional endocardial motion estimation from electro-anatomical mapping data: Application to scar characterization," IEEE Trans. Biomed. Eng, vol. PP, no. 99, p. 1, 2012.

[7] C. Ghule et al., "Design of portable arm processor based ecg module for 12 lead ecg data acquisition and analysis," in ICBPE 2009, Dec 2009, pp. $1-8$.

[8] P. Uusimaa et al., "Collagen scar formation after acute myocardial infarction: Relationships to infarct size, left ventricular function, and coronary artery patency," Circ., vol. 96, no. 8, pp. 2565-2572, 1997.

[9] R. H. Selvester et al., "A digital computer model of the vectorcardiogram with distance and boundary effects: Simulated myocardial infarction," Am Heart J., vol. 74, no. 6, pp. 792 - 808, 1967.

[10] D. Romero et al., "Depolarization changes during acute myocardial ischemia by evaluation of qrs slopes: Standard lead and vectorial approach," IEEE Trans. Biomed. Eng, vol. 58, no. 1, pp. $110-120$, Jan 2011.

[11] J. Rosengarten et al., "Can QRS scoring predict left ventricular scar and clinical outcomes?" Europace, in press, doi:10.1093/europace/eut014.

[12] S. M. Narayan et al., "Relation of t-wave alternans to regional left ventricular dysfunction and eccentric hypertrophy secondary to coronary heart disease," Am J Cardiol, vol. 97, no. 6, pp. 775 - 780, 2006.
[13] P. de Chazal et al., "Automatic classification of heartbeats using ECG morphology and heartbeat interval features," IEEE Trans. Biomed. Eng., vol. 51, no. 7, pp. $1196-1206$, Jul 2004.

[14] V. Bono et al., "Development of an Automated Updated Selvester QRS Scoring System using SWT-based QRS fractionation detection and classification," IEEE Journal of Biomedical and Health Informatics, vol. DOI:10.1109/JBHI.2013.2263311,(in press).

[15] E. Mazomenos et al., "A time-domain morphology and gradient based algorithm for ecg feature extraction," in IEEE ICIT, Mar 2012, pp. 117 -122 .

[16] D. Kugiumtzis et al., "Measures of analysis of time series (mats): A matlab toolkit for computation of multiple measures on time series data bases," Journal of Statistical Software, vol. 33, no. 5, pp. 1-30, 2010.

[17] K. Saiveena et al., "Characterization of ecg signals using multiscale approach," in Signal Processing and Communications (SPCOM), 2012 International Conference on, 2012, pp. 1-5.

[18] A. Noviyanto et al., "Selecting features of single lead ecg signal for automatic sleep stages classification using correlation-based feature subset selection," International Journal of Computer Science Issues (IJCSI), vol. 8, no. 5, p. 139, 2011.

[19] T. Penzel et al., "Comparison of detrended fluctuation analysis and spectral analysis for heart rate variability in sleep and sleep apnea," IEEE Trans. Biomed. Eng, vol. 50, no. 10, pp. 1143 -1151, 2003.

[20] M. Singh, Introduction to Biomedical Instrumentation. Prentice Hall of India, 2010.

[21] E. Frank, "An accurate, clinically practical system for spatial vectorcardiography," Circ., vol. 13, no. 5, pp. 737-749, 1956.

[22] G. Dower et al., "On deriving the electrocardiogram from vectoradiographic leads." Clin Cardiol, vol. 3, no. 2, pp. 87-95, apr 1980, review,

[23] A. L. Goldberger et al., "PhysioBank, PhysioToolkit, and PhysioNet: Components of a New Research Resource for Complex Physiologic Signals," Circ., vol. 101, no. 23, pp. 215-220, 2000.

[24] A. Christmann et al., Support Vector Machines, ser. Information Science and Statistics. Springer, 2008.

[25] L. Rokach et al., Data mining with decision trees : theory and applications. Hackensack, N.J. : World Scientific, 2008.

[26] A. Elisseeff et al., "Leave-one-out Error and Stability of Learning Algorithms with Applications," in Learning Theory and Practice, ser. NATO ASI Series. Amsterdam; Washington, DC: IOS Press, 2002.

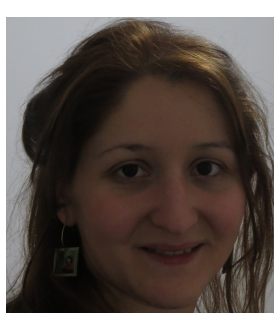

Sofia Maria Dima is a Ph.D. candidate at the Electrical and Computer Engineer Department in the University of Patras (UoP). Sofia has received her Electrical and Computer Engineer Diploma from the same Department of UoP in 2010. Her main research interests include Wireless Sensor Networks, Data Mining, Signal Processing, Task Scheduling and Embedded Systems. Sofia has worked as a researcher in EU-funded R\&D projects and as a teaching assistant.

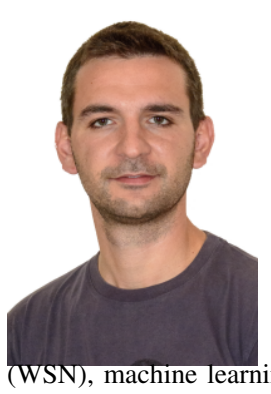

Christos Panagiotou received his 5-year Diploma degree in Computer Engineering and Informatics from the Univ. of Patras in 2007. He has been involved in research projects funded by the European Union and Greek organizations as a research fellow of the Industrial Systems Institute / ATHENA RC from 2009. Currently he is a $\mathrm{PhD}$ student of Electrical and Computer Engineering, Univ. of Patras.

His research interests are in the area of energy efficient middlewares for Wireless Sensor Networks $\mathrm{g}$ and data mining for biomedical applications. 


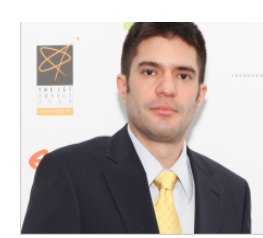

Evangelos B. Mazomenos received a Diploma degree (5-year) in Electrical \& Computer Engineering from the Univ. of Patras, Greece and a PhD from the School of ECS, Univ. of Southampton, UK in 2006 and 2012 respectively. He is the recipient of the 2009 IET Leslie H. Paddle fellowship on postgraduate studies for his $\mathrm{PhD}$ research on real-time target tracking in WSN. From January 2011 he has been a research fellow in ECS.

His research interests are in the area of WSNs with a focus on positioning and tracking, biomedical signal processing and dynamic estimation algorithms.

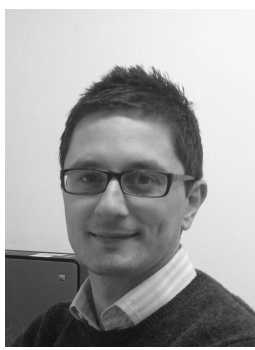

James Rosengarten received his MBBS from the University of London in 2002 and became a member of of the Royal College of Physicians (MRCP(UK)) in 2005. Currently he is a clinical cardiologist based in Southampton. Following training in general cardiology in London and Wessex, he is now specialising in heart rhythm disorders, including invasive testing and implantable devices. He is currently the Research Fellow in Cardiac Rhythm Management, based at University Hospital Southampton. Under the supervision of Prof. Morgan and Prof. Hanson at the University of Southampton, he is utilising engineering techniques to discover novel biomarkers of sudden cardiac death risk.

Koushik Maharatna (MâĂŹ02) received the M.Sc. degree in electronic science from Calcutta Univer-

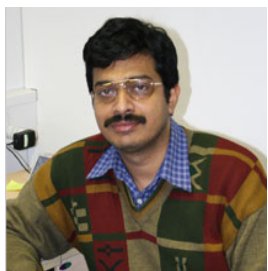
sity, Calcutta, India, in 1995 and the Ph.D. degree from Jadavpur University, Calcutta, India, in 2002. From 1996 to 2000, he was involved in different projects sponsored by the Government of India undertaken at the Indian Institute of Technology (IIT), Kharagpur, India. From 2000 to 2003, he was a Research Scientist in IHP, Frankfurt (Oder), Germany. During this phase, his main involvement was related to the design of a single-chip modem for the IEEE 802.11a standard. In September 2006, he joined the School of Electronics and Computer Science of the University of Southampton, U.K., where he is currently a Reader. His research interests include low-power VLSI and signal processing for applications in DSP, communication and nextgeneration healthcare systems, computer arithmetic, analog signal processing, and bio-inspired circuits and systems.

Dr. Maharatna is a member of the IEEE VLSI System Application (VSA) Technical Committee.

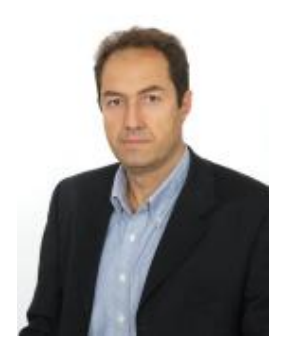

John Gialelis John V. Gialelis received the Ph.D degree in electrical engineering from the University of Patras, the M.Sc degree in electrical engineering from the Polytechnic Institute of New York University (NYU Poly), U.S and the B.Sc in electrical engineering from Fairleigh Dickinson University, U.S. Since 1996 he has been a research fellow and a lecturer in the School of Engineering at the University of Patras, Greece and he is a member of the Industrial Systems Institute / ATHENA RC. He has authored or co-authored more than 50 articles in refereed journals, conference proceedings and book chapters and supervised many research projects funded by the European Union and Greek organizations. His research interests include networking of embedded systems, semantic enrichment of sensor data, pattern mining and ontology engineering.

$\mathrm{He}$ is a regular reviewer in many scientific journals. He is a member of the IEEE Industrial Electronics Society.

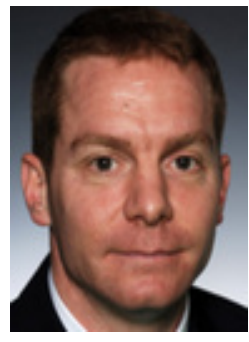

Nick Curzen is a Consultant Cardiologist at University Southampton Hospitals and Professor of Interventional Cardiology at the University of Southampton. Betwenn 1993-1996 he trained in cardiology at Southampton, Bournemouth, Royal Brompton, London Chest and St Bartholomews hospitals and completed a $\mathrm{PhD}$ in vascular biology at Imperial College, London. He has wide ranging research interests including platelet function, myocardial ischaemia, cardiac magnetic resonance and scar. $\mathrm{He}$ has over 130 peer review scientific papers and has edited 3 cardiology textbooks.

In November 2010, Nick Curzen came top of a national poll of interventional cardiologist published in the Daily Mail.

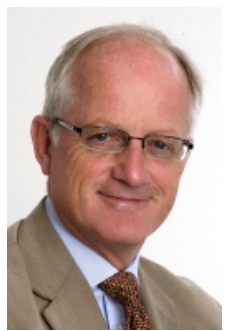

John Morgan graduated from the University of Cambridg with a BA in Natural Sciences in 1979 and from the Westminster Medical School with an MB BChir in 1982. He then obtained an MA and an MD from Cambridge University in 1983 and 1991 respectively. He became a member of the Royal College of Physicians (MRCP(UK)) in 1985 and Fellow (FRCP) in 1995. On his appointment as Consultant Cardiologist, Wessex Cardiothoracic Centre (1992), he founded the Wessex Cardiac Arrhythmia Management Service which has become recognised as a centre of clinical and research excellence. He was appointed an honorary professor at the University of Teesside in 2006 for his work in medical education and received a personal chair in the School of Medicine, University of Southampton in 2007. Professor Morgan holds several published patents relating to invention of novel interventional and device technologies.

He supervises/has supervised several fellows in doctoral research projects and continues to research and publish extensively and is an internationally recognised leader in his field. He also sits on a series of national and international boards/professional associations and governing bodies. 